\title{
Efficacy of Sandostatin LAR in Type 1 DM; Improvement in Glycemic Control with less Insulin. A Case Report
}

\author{
James Bonucchi DO${ }^{1}$, Kathy Lilli RN ${ }^{1}$, Udaya Kabadi ${ }^{1,2, *}$ \\ ${ }^{1}$ University of Iowa Hospitals and Clinics, Iowa City, IA. ${ }^{2}$ VA Medical Center, Des Moines, IA, udaya.kabadi@va.gov
}

Received 10 January 2011; revised 13 February 2011; accepted 21 February 2011.

\begin{abstract}
Objective: Octreotide three times daily is reported to reduce daily insulin by $50 \%$ in patients with Type 1 DM. Therefore, we assessed the impact of long acting Octreotide (Sandostatin LAR) monthly intramuscular administration in a subject with Type 1 DM. Methods: A 32-year-old man with Type 1 DM of 16 years participated after obtaining informed consent. He had no microvascular or macrovascular complications. He continued the present insulin regimen for four weeks. IM Sandostatin LAR $20 \mathrm{mg}$ was initiated and increased at four weeks to $30 \mathrm{mg}$. He was followed every four weeks for six months. Insulin regimen was adjusted every one to two weeks based on blood glucose before meals, bedtime and on onset of hypoglycemic symptoms. He continued other medications, previous diet and activity. Assessment of HbA1c, serum electrolytes, urea nitrogen, creatinine, TSH, free T4, liver enzymes, complete blood cell counts, vitamin B12, lipids and insulin regimen were performed at the initiation and end of the study. Results: HbA1c declined from 9 to $8 \%$ with reduction in daily insulin dose from 55 to 43 units, with a major reduction in insulin Glargine, 50 to 40 units. Body weight remained unaltered. Other laboratory tests including gallbladder examination remained unchanged Conclusion: Monthly Sandostatin LAR administration may improve glycemic control with less insulin in Type 1 DM.
\end{abstract}

Keywords: Type 1 Diabetes Mellirtus, Sandostatin LAR, Glycemic Control, Daily Insulin Dose

\section{INTRODUCTION}

Short acting Octreotide has been demonstrated to render uniformity to diurnal glycemia in Type 1 diabetes

The study was conducted with research grant by Novartis Pharmaceuticals Inc. It was presented at Endocrine Society Annual Meeting in June 2008. mellitus via inhibition of glucagon and human growth hormone as well as reduction of carbohydrate absorption [1-8]. Therefore, long acting octreotide (Sandostatin LAR) is likely to exert a similar effect in type 1 diabetes mellitus. However, Sandostatin LAR has not been assessed in this regard. Herein, in this report, we describe a patient with type $1 \mathrm{DM}$ in whom a short treatment with Sandostatin LAR improved glycemic control while requiring less insulin.

\section{A Case Report}

Treatment with Sandostatin LAR was approved by the local Institutional Review Board. 32-year-old man with Type 1 DM completed the treatment with Sandostatin LAR for 28 weeks. Type 1 diabetes was previously confirmed with presence of GAD antibody and absence of C-peptide in the serum. He has no history of diabetes complications. He continued to receive his prior insulin regimen for four weeks and then IM Sandostatin LAR was added to his regimen in the same prescription as used in patients with acromegaly [9] Thus, the initial dose of Sandostatin LAR was $20 \mathrm{mg}$. He was followed at intervals of 4 weeks throughout the treatment period lasting 28 weeks. The dose of Sandostatin LAR was increased to $30 \mathrm{mg}$ after 4 weeks. During this time, his insulin regimen was adjusted over the telephone as required at intervals of every 1-2 weeks based on his self blood glucose records. He continued oral medications for other disorders throughout the period. Diet and activity profile were maintained throughout the treatment as well. He monitored his blood glucose pre meals and at bedtime as well as at the onset of symptoms of hypoglycemia, i.e., confusion, extreme sweating, dizziness, palpitation etc. The presence of hypoglycemia was confirmed by blood sugars of less than $70 \mathrm{mg} / \mathrm{dl}$ recorded in his diary. We also assessed pre-meal, $2 \mathrm{hr}$ post-meal, bedtime and 3 am blood sugar levels for 24 hrs on two occasions, immediately prior to initiation of Sandostatin LAR and at the end of treatment period. An ultrasound of the gallbladder was performed prior to initiation of Sandostatin LAR and at the end of the treatment. Body 
Table 1. HbA1c, daily dosage of insulin glargine, aspart and total insulin, and hypoglycemic episodes (blood sugar less than 70 $\mathrm{mg} / \mathrm{dl}$ ) during the two weeks prior to initiation(A) with sandostatin LAR and at the end of the treatment period (B).

\begin{tabular}{ccccccc}
\hline & & HbA1c (\%) & Insulin Glargine (units/day) & Insulin Aspart (units/day) & Total insulin dose (units/day) & Hypoglycemic events \\
\hline A & A & 9 & 50 & 45 & 5 & 95 \\
B & B & B & 8 & 40 & 39 & 79 \\
\hline
\end{tabular}

weight, HbA1c, serum electrolytes, creatinine, urea nitrogen, TSH, free thyroxine, liver enzymes, complete blood cell counts, vitamin B12 and lipid profile were determined at 12 weeks after initiation and at the end of the treatment period. Comparisons were made for body weight, HbA1c, daily insulin dose, and hypoglycemic episodes documented during 2 weeks prior to initiation of Sandostatin LAR and at the end of the treatment period.

\section{Results}

The patient tolerated Sandostatin LAR well although required pancreatic enzyme replacement (Creon 10) intermittently to prevent diarrhea upon consumption of a high fat meal. An 11\% reduction in HbA1c was noted while requiring $17 \%$ reduction in total daily insulin dose at the end of this treatment with Sandostatin LAR (Table 1), whereas, the remainder of his laboratory tests and ultrasound examinations remained unchanged.

\section{Discussion}

Octreotide acetate is a synthetic, long acting, octapeptide analogue of the natural hormone, somatostatin. [1] Like somatostatin, Octreotide exerts inhibitory effects on the release of pituitary and gastroenteropancreatic hormones (i.e. GH, TSH, insulin, glucagon, CCK, VIP and gastrin) inhibits gastric acid, pancreatic enzyme secretion and bile flow; prolongs intestinal transit time, and decreases gallbladder contractility[5-8,10]. Octreotide is a specific and potent somatostatin-receptor type 2 agonist. Compared to native somatostatin, Octreotide is 45 times more potent in terms of inhibition of growth hormone (GH) secretion, 11 times more potent in inhibition of glucagon, but only 1.3 times as active in inhibition of insulin secretion[5].

A reduction in daily insulin dose of up to $50 \%$ was required in many Octreotide-treated insulin-dependent diabetics [1-4] and physiologically, Sandostatin LAR may affect glucose regulation in a similar manner. Therefore, hypoglycemic episodes may occur 10-14 days after the initial Sandostatin LAR injection if insulin dose is not reduced especially in patients with desirable glycemia. This report demonstrates that Sandostatin LAR administration in a patient with Type 1 diabetes improved glycemic control while requiring less insulin to avoid hypoglycemia without weight gain. This outcome may be attributed to its inhibitory action on growth hormone and glucagons [5-7].

\section{Conclusion}

This preliminary study suggests that Sandostatin LAR may improve glycemic control while decreasing daily insulin dose in patients with Type 1 diabetes without weight gain.

\section{REFERENCES}

[1] Dimitradis, G., et al. (1983) Effects of long acting somatostatin analogue on post-prandial hyperglycemia in insulin-dependent diabetes mellitus. Metabolism 32, 987992. doi:10.1016/0026-0495(83)90140-3

[2] Spinas, G.A., et al. (1985) .Reduced post-prandial hyperglycemia after subcutaneous injection of a somatostatinanalogue (SMS 201-995) in insulin dependent diabetes mellitus. Diabetes Care, 8, 429-435. doi:10.2337/diacare.8.5.429

[3] Rios, M.S., Navascues, I., Saban, J., Ordonez, A., Sevilla, F. and Del Pozo, E. (1986) Somatostatin analog SMS 201-995 and insulin needs in insulin-dependent diabetic patients studied by means of an artificial pancreas. The Journal of Clinical Endocrinology \& Metabolism, 63, 1071-1074.

[4] Grossman, L.D., Shumak, S.L., George, S.R., Singer, W. and Zinman, B. (1989) The effects of SMS 201-995 (sandostatin) on metabolic profiles in insulin-dependent diabetes mellitus. The Journal of Clinical Endocrinology \& Metabolism, 68, 63-70. doi:10.1210/jcem-68-1-63

[5] Ludwigsen, E., Stridsberg, M., Taylor, J.E., Cutler, M.D., Oberg, K., Janson, E.T. and Standler S. (2007) Regulation of insulin and glucagon secretion from rat pancreatic islets in vitro by somatostatin analogues. Regulatory Peptides, 138, 1-9. doi:10.1016/j.regpep.2006.07.006

[6] Singh, V., Brenel, M.D., Zacharias, S., Mergler, S., Jahr, H., Wiedemnan, B., Bretzel, R.G., Plockinger, U., and Strowski, M.Z., (2007) Charecterization of soamtostatin receptor subtype-specific regulation of insulin and glucagon secretion: An in vitro study on isolated human pancreas. The Journal of Clinical Endocrinology \& Metabolism, 92, 673-680. doi:10.1210/jc.2006-1578

[7] Brazeau, P., Vale, W. and Burgus, R. (1973) Hypothalamic Polypeptide that Inhibits the Secretion of Immunoreactive Pituitary Growth Hormone. Science, 179, 77-83. doi:10.1126/science.179.4068.77

[8] Fery, F., Tappy, L., Schneiter, P., Daviere, J.E. and Balasse, O. (2005) Effect of Somatostatin on Duodenal Glucose Absorption in Man. The Journal of Clinical Endocrinology \& Metabolism, 90, 4163-4169. doi:10.1210/jc.2004-1766

[9] Manjila, S., Wu, O.C., Khan, F.R., Khan, M.M., Arafah, B.M. and Setman, W.R. (2010) Pharmacological management of acromegaly: A current perspective. Neurosurgical Focus, 29, E14-24. doi:10.3171/2010.7.FOCUS10168

[10] Corleto, V.D. (2010) Somatostatin and the gastrointestinal tract. Current Opinion in Endocrinology, Diabetes \& Obesity, 17, 63-68. 